\title{
Brief Communication: Measuring rock decelerations and rotation changes during short-duration ground impacts
}

\author{
Andrin Caviezel ${ }^{1}$ and Werner Gerber ${ }^{2}$ \\ ${ }^{1}$ WSL Institute for Snow and Avalanche Research SLF, 7260 Davos Dorf, Switzerland \\ ${ }^{2}$ Swiss Federal Institute for Forest, Snow and Landscape Research WSL, 8903 Birmensdorf, Switzerland
}

Correspondence: Andrin Caviezel (andrin.caviezel@slf.ch)

Received: 28 March 2018 - Discussion started: 16 May 2018

Accepted: 13 November 2018 - Published: 23 November 2018

\begin{abstract}
Rockfall trajectories are primarily influenced by ground contacts, causing changes in acceleration and rock rotation. The duration of contacts and its influence on the rock kinematics are highly variable and generally unknown. The lack of knowledge hinders the development and calibration of physics-based rockfall trajectory models needed for hazard mitigation. To address this problem we placed three-axis gyroscopes and accelerometers in rocks of various sizes and shapes with the goal of quantifying rock deceleration in natural terrain. Short ground contacts range between 8 and $15 \mathrm{~ms}$, longer contacts between 50 and $70 \mathrm{~ms}$, totalling to only $6 \%$ of the runtime. Our results underscore the highly nonlinear character of rock-ground interactions.
\end{abstract}

\section{Introduction}

A detailed understanding of object penetration into matter is essential from both a fundamental physics and geophysical point of view. The relevant timescale spans from highspeed impacts of kilometers per second in planetary science to centimeters per second or millimeters per second in laboratory experiments of intruder sinking into granular beds. There are many studies on penetration of objects into granular media or coefficients of restitution (see Altshuler et al., 2014; Asteriou et al., 2012, and references therein). However, there is little understanding of processes arising from altered impact conditions, such as deviations from normal impact configurations, high rotational speeds of the impacting object, etc. Consequently, the understanding of the mechanics of rock-ground interactions poses a longstanding problem in rockfall engineering. This interaction defines the speed, jump height and dispersion of falling rocks in natural terrain. Because ground interaction controls rockfall runout distances and energy levels, it is the core problem when developing physics-based dynamic models for rockfall hazard mitigation and planning (Leine et al., 2014).

One approach to address the impact problematic is to use dendrogeomorphic techniques to asses rockfall frequency and distribution (Trappmann and Stoffel, 2015; Corona et al., 2017) and/or trajectory reconstruction via impact analysis (Paronuzzi, 2009; Saroglou et al., 2018). Hardly any data exist that directly measure rock-ground interactions during a rockfall event. A possible method to characterize ground impacts is to study surface scars left by falling rocks. For example, translational rock velocities can be determined by measuring the distance between ground contacts and the relative slope angle between the contact points. For an initial estimation it can be assumed that the jump height will be about $1 / 10$ of the jump distance on the slope. Based on such an assumption, a flight parabola is determined and the relevant velocities can be calculated (Gerber, 2015). If different jump heights are assumed, e.g., $1 / 8$ or $1 / 12$, the maximum velocities shortly before ground impact will change by less than $10 \%$. In many cases, this method suffices to obtain a rough estimate of the dissipative character of the ground interaction.

The problem with many approaches is that ground scarring is often difficult to physically interpret, especially if the rock is in a fast rotating, rolling motion. In this case the distances between ground contacts are extremely short and provide little information concerning the true velocity of the rock. Although the depth of the ground scar is an indication of the rebound mechanics at work, scar depths are highly variable, 
especially if the rock is "skipping" on the ground surface. Moreover, the analysis of rockfall traces provides little information of the mechanics of ground interaction, particularly if the relationship between the translational and rotational kinematics of the rock are unknown.

Newer studies in penetration studies make use of emerging microelectronic mechanical sensors to directly track the occurring motion (Sanchez-Colina et al., 2014; Gronz et al., 2016). Note that to date the major drawback of available multi-degree-of-freedom inertial measurements units (IMUs) is the range restriction to low accelerations (few tens of $g$ ). Because the major application for such IMUs is unmanned aerial vehicle (UAV) flight control, resistance to and measurement capabilities of heavy impacts is not the main focus of chip makers.

In this paper, we present novel and detailed in situ measurements of high-impact ground interaction contact times, decelerations and changes in rock rotations using sensors inserted inside the rock. The resulting three-dimensional measurements yield detailed insights into how rocks behave, both in flight and upon contact with the ground. The measurements guide towards how experimental field campaigns can be constructed to obtain the necessary data needed to calibrate constitutive relationships for dynamic rockfall models.

However, before any conclusions can be reached or any further calculations made based on the results, the measurements must be subjected to a quality check and verified. At present we have little idea of the degree of acceleration reversal and change of rotational speed during impact, making it difficult to judge the accuracy of the measurements. Simple kinematic requirements must be fulfilled. For example, the acceleration measurements at rest must correspond to the value of gravitational acceleration and indicate a value of zero in free flight. The purpose of this brief communication is therefore to elaborate on measurement frequencies and methods needed to capture the physical information required to study rock-ground interaction in natural terrain. We believe this information is necessary to develop better trajectory models for rockfall hazard mitigation.

\section{Methods of measurement and evaluation}

\section{$2.1 \quad$ Field studies}

The rockfall tests were performed in natural terrain. The test site, located near Tschamut in the canton of Grisons, is a slope $50 \mathrm{~m}$ high with a maximum inclination of $42^{\circ}$, running down to a horizontal surface. The surface vegetation consists mainly of grass, with a few scattered shrubs in the upper, steeper part of the slope. The absence of tall vegetation and relatively smooth terrain allow a clear observational view and make the Tschamut site ideal for conducting rockfall experiments and filming the rocks' movements. A release point at the top of the slope was selected, measured and used
Table 1. Mean values of absolute rotational and acceleration data for calculating eccentricities.

\begin{tabular}{lrrr}
\hline Time $(\mathrm{s})$ & $59.0-59.7$ & $59.9-61.0$ & $61.2-61.9$ \\
\hline Rotation $\left({ }^{\circ} \mathrm{s}^{-1}\right)$ & 3690 & 3501 & 4098 \\
Acceleration $\left(\mathrm{m} \mathrm{s}^{-2}\right)$ & 16.20 & 14.01 & 18.9 \\
Eccentricity $(\mathrm{m})$ & 0.004 & 0.004 & 0.004 \\
Eccentricity $(\mathrm{mm})$ & 3.91 & 3.75 & 3.70 \\
\hline
\end{tabular}

to release the rocks by simple dropping (no or little initial translational velocity and initial spin). The release point was selected to accommodate the transport of rocks, facilitating experimental data sets of more than 50 releases on a single day (i.e., with the same ground conditions including temperature and moisture content).

We present the results of one out of more than 50 trajectories captured in a test series specifically designed to investigate the role of rock shape on runout and dispersion; see Caviezel et al. (2018c, a). In this particular measured run, an artificially manufactured concrete block with an $0.3 \mathrm{~m}$ edge length and a mass of $44 \mathrm{~kg}$ was released. The symmetric and well-defined block shape was used as a control geometry in the rockfall experiments. The rock's corners and edges were pared back a quarter to make the block less dice shaped. A hole $68 \mathrm{~mm}$ in diameter was drilled through the block to accommodate the sensor. The block's mass and volume $\left(0.019 \mathrm{~m}^{3}\right)$ make it equivalent to a sphere with a radius of $0.165 \mathrm{~m}$ and a circumference of $1.04 \mathrm{~m}$ with a rock density of $2315 \mathrm{~kg} \mathrm{~m}^{-3}$.

\subsection{Sensor}

In view of developments in consumer electronics for devices including tablets, mobile phones and UAVs, the measurement ranges and performances of available miniaturized motion sensors are steadily increasing. In situ data were recorded using a dedicated low-power sensor node, dubbed StoneNode (Fig. 1a); see Caviezel et al. (2018b). The main components of StoneNode v1.0, which was used to record the data presented here, are a triaxial accelerometer with a measurement range of $400 \mathrm{~g}$ and an InvenSense three-axis gyroscope recording up to $4^{\prime} 000^{\circ} \mathrm{s}^{-1}\left(22.2 \pi \mathrm{rad} \mathrm{s}^{-1}\right)$. Analysis of frequency measurements yielded values of $400 \mathrm{~Hz}$ during acceleration and $487.5 \mathrm{~Hz}$ for rotation. A microcontroller manufactured by Texas Instruments hosts the sensors and was selected for its low power consumption (roughly $3.6 \mathrm{~mW}$ at $3 \mathrm{~V}$ ). Thus, a $1100 \mathrm{~mA} \mathrm{~h} \mathrm{LiPo} \mathrm{battery} \mathrm{can}$ gather $56 \mathrm{~h}$ of data. Efficient data retrieval is ensured using a plug-and-play USB device. For detailed information on the sensors used and comparison with other systems, see Niklaus et al. (2017) and Caviezel et al. (2018c, b). 
(a)

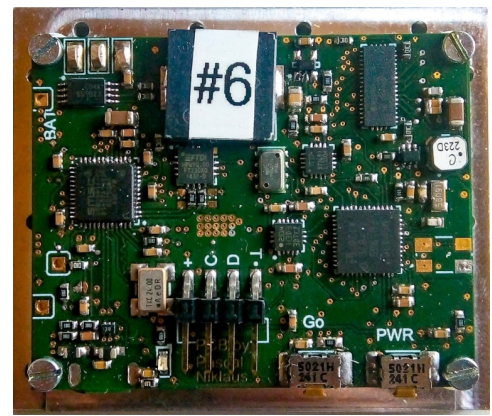

(c)

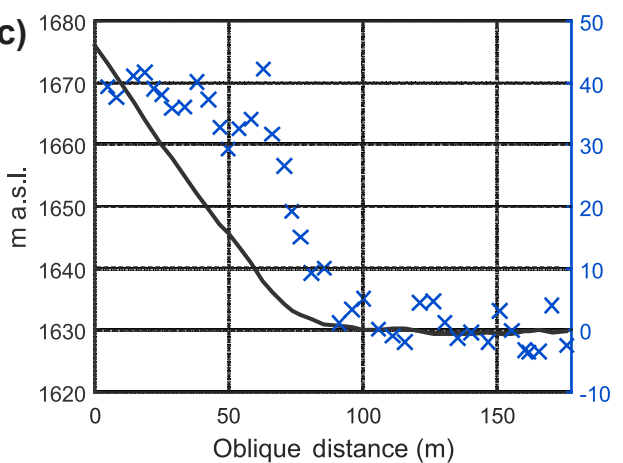

(b)
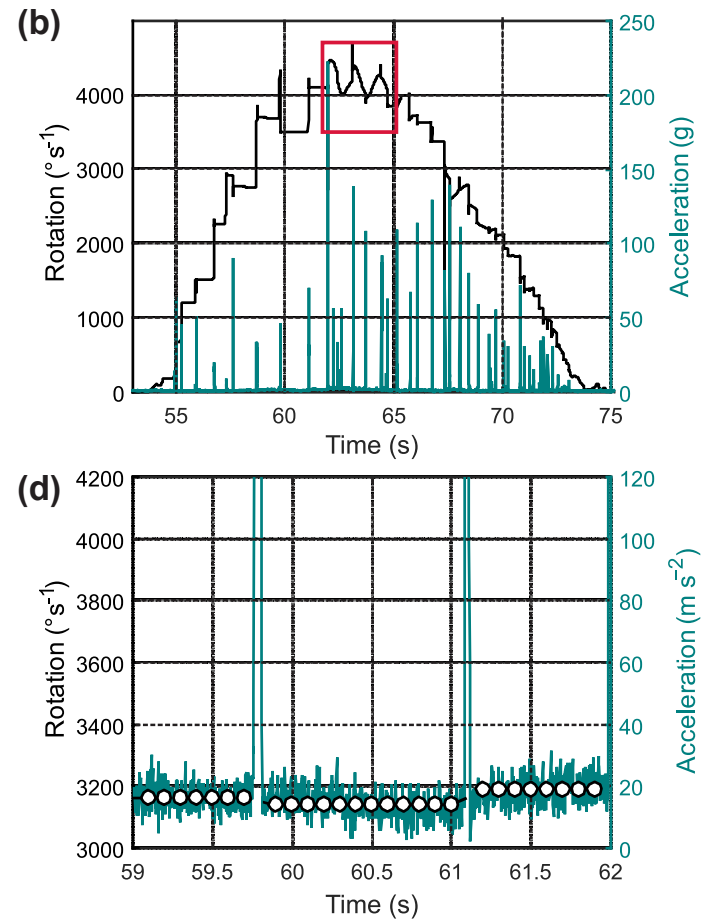

Figure 1. (a) An exposed micro-controller board hosting all the MEMS sensors, microSD card and a USB connector powered by a $1100 \mathrm{~mA} h$ battery (both covered by the board). (b) Sensor data stream showing absolute rotational velocities and acceleration values during the $20 \mathrm{~s}$ movement phase (from 54 to $74 \mathrm{~s}$ ), the rectangle indicating range with one saturated axis. (c) Slope distance of the projected trajectory of the stone with its location and slopes. (d) Absolute rotational velocities and acceleration values, plus mean acceleration values within the intervals of impacts (white dots) for calculating eccentricity.

\subsection{Quality analysis}

Before the measurements can be processed, the raw data need to be verified. Assuming that the sensors are functioning properly, the raw data should be checked for the following criteria:

- The measuring range of each individual sensor should not be exceeded.

- When at rest, the rotational velocity should equal zero and the acceleration values should equal 1 , corresponding to gravitational acceleration.

- During free fall, the rotational velocity should remain constant, with zero absolute acceleration; this analysis must be performed when there is relatively little rotation, the influence of centripetal acceleration will grow at higher rotational velocities.

- Theoretically, when rotational velocity is constant, if there is an offset with the sensors and the center of mass, the phenomenon of centripetal acceleration should result in the measurement of higher values.

During free fall, the rotational velocities and acceleration values can then be correlated, representing the eccentrically fitted sensor as the rock's center of gravity. In physical terms, this relationship can be expressed by the formula Eq. (1):

$R_{\mathrm{e}}=\frac{a_{Z}}{\omega^{2}}$,

where $R_{\mathrm{e}}$ is the sensor's eccentricity $(\mathrm{m}), a_{Z}$ the centripetal acceleration $\left(\mathrm{m} \mathrm{s}^{-2}\right)$ and $\omega$ angular velocity $\left(\mathrm{rad} \mathrm{s}^{-1}\right)$. In theory, rotational differences should result in the same eccentricities.

\section{Results}

\subsection{General}

The raw data comprise measurements starting from when the sensor was switched on until the block's deposition some $74 \mathrm{~s}$ later. The effective start of the rockfall occurred after around $54 \mathrm{~s}$. During this period, 8000 acceleration values for all three axes ( $x, y$ and $z$ ) and 9750 rotational values were measured. During the $20 \mathrm{~s}$ rockfall from release to deposition, the block covered a horizontal distance of $147 \mathrm{~m}$ and negotiated a height difference of $49 \mathrm{~m}$. The maximum inclination on site was $-42^{\circ}$, dropping to zero and even $+4^{\circ}$ on the upslope of the depositional area. The effective fall trajectory's slope length was $162 \mathrm{~m}$ (Fig. 1c). 
Ground contacts are very clearly indicated by sharp peaks in the acceleration measurements and changes in rotational velocities. In steeper terrain, higher velocities and larger jump lengths lead to significantly fewer ground contacts than in the runout zone with roll-out behavior. Absolute rotation increases from an initial value of zero to $4^{\prime} 500$ degrees per second $\left({ }^{\circ} \mathrm{s}^{-1}\right)$ before falling back to zero.

The maximum absolute acceleration value measured was $225 g$ (at second 62.0). The rest of the measured values range below $140 \mathrm{~g}$, and many were even less than $50 \mathrm{~g}$ (Fig. 1b).

\section{Quality analysis procedure}

The absolute rotational velocities and acceleration values were calculated and presented in the preceding section. The peak values of the individual measurements were checked. Due to the symmetry braking caused by the sensor hole, a main rotational axis exists that reaches the saturation limit between 62 and $64.6 \mathrm{~s}$. This causes the resultant trace to predominantly feature the oscillating mode of the two remaining axes highlighted with the box in Fig. $1 \mathrm{~b}$.

The sensors are specified to high-resolution capabilities of $0.122^{\circ} \mathrm{s}^{-1}$ in the case of the gyroscope and $0.195 \mathrm{~g}$ for the accelerometer. These values hold only when maximum sensitivity settings are used. In the used case, full-scale range is needed for both sensors; thus the measured deviations increase significantly. As described in Niklaus et al. (2017) these main deviations can be corrected via a linear correction function $f(x)=c_{0}+c_{1} \cdot x$ for each sensor axis. If applied, $c_{0}$ is the dominant correction term for the accelerometer in the order of 0.15 to $3 \mathrm{~g}$. For the gyroscope, $c_{1}$ is dominant, being of the order of $0.09 \%$ to $0.35 \%$ from the ideal value of 1.0 . Note that the measured offsets lie below $1 \%$ of the full-scale range and thus can be neglected. For the presented trajectory the actual sensor offset at rest amounts to $4.5 \pm 0.1^{\circ} \mathrm{s}^{-1}$ and $1.17 \pm 0.48 \mathrm{~g}$ as opposed to the ideal value of $1 \mathrm{~g}$.

The free-fall analysis of the measurements began immediately after the initial motion, at $54.5 \mathrm{~s}$. At this time its rotation is relatively low $\left(180.5^{\circ} \mathrm{s}^{-1}\right)$ and its influence on the acceleration value small. Equation (1) yields

$a_{Z}=R_{\mathrm{e}} \cdot \omega^{2}=0.165 \cdot\left(\frac{180.5}{360} \cdot \pi\right)^{2}=0.41 \mathrm{~m} \mathrm{~s}^{-2}$.

Eccentricity was analyzed between 59.0 and $61.9 \mathrm{~s}$, ignoring data from the two intervening ground contacts (Fig. 1d). The mean acceleration values within these three intervals are used to feed Eq. (1) and to determine the eccentricity radius, which is virtually identical for all three time intervals, equalling $0.004 \mathrm{~m}$ (Table 1).

\subsection{Duration of ground contacts}

Ground contacts are clearly recognizable from the measured rotational and acceleration values. Very short ground contacts last $8-15 \mathrm{~ms}$, medium-length contacts $20-40 \mathrm{~ms}$ and lengthy contacts $50-75 \mathrm{~ms}$. During the first $2-3 \mathrm{~s}$ after the rock has been set in motion, the duration of ground contacts increases very quickly to the peak values and then drops back to values of $10-30 \mathrm{~ms}$, remaining at this level on flat terrain (Fig. 2a). This corresponds to the intuitive understanding of more excessive scarring, that is deeper and longer ground penetration, of rocks with higher kinetic energy for a given soil softness. Remarkably, the total contact times that determine the trajectory kinematics amount only to $14 \%$ of the total trajectory time of $21 \mathrm{~s}$, or if the roll-out section after the last recorded impact is excluded, to only $6 \%$ of the total runtime.

\subsection{Details of individual ground contacts}

Individual results on absolute rotational and acceleration values during ground contacts are presented below. Here, we classified a contact as the temporal evolution between two plateaus in angular velocity. A typical contact during the acceleration phase is shown in Fig. 2b. Rotational velocities increase with almost every - relatively short - ground contact, as at $55.24 \mathrm{~s}$. This contact lasted $42 \mathrm{~ms}$ at a maximum acceleration of $45.6 \mathrm{~g}$ and increased rotation from 683 to $1^{\prime} 087^{\circ} \mathrm{s}^{-1}$. This typical behavior in steep terrain implies that the rotation change is a function of the inclination of the slope. Here, the rotation increases with slope angles higher than $38^{\circ}$ and decreases under an inclination lower than $20^{\circ}$.

The ground contact featured in Fig. 2c (which lasted $28 \mathrm{~ms}$, starting at $57.62 \mathrm{~s}$ ) exhibits larger accelerations of $90.0 \mathrm{~g}$ while the rotation tipped from $2^{\prime} 921$ to $2^{\prime} 766^{\circ} \mathrm{s}^{-1}$, indicating an opposed faced obstacle within the acceleration path. Both ground contacts shown above have clear maxima in the accelerometer data. However, some contacts with two or even more maxima were also recorded. A relatively long ground contact occurred at $58.72 \mathrm{~s}$, lasting $68 \mathrm{~ms}$ (Fig. 2d). During this time, two main acceleration maxima were measured: 33.4 and $30.4 \mathrm{~g}$, respectively. During this ground contact, rotation increased steadily from 2758 to $3696^{\circ} \mathrm{s}^{-1}$.

If the angular velocity between two acceleration peaks remains constant, neither steadily rising nor falling, it indicates that two separate ground contacts occur, similar to those occurring at 63.13 and $63.15 \mathrm{~s}$ shown in Fig. 2e. Here, the contact times are very short (lasting 13 and $8 \mathrm{~ms}$ ) and the acceleration maxima differ (138.6 and $34.3 \mathrm{~g}$ ). During these two contacts, rotation increased from $4^{\prime} 186$ to $4^{\prime} 387^{\circ} \mathrm{s}^{-1}$, with a constant intermediate value to $4^{\prime} 334^{\circ} \mathrm{s}^{-1}$. Interestingly, the maximum rotation of $4^{\prime} 709^{\circ} \mathrm{s}^{-1}$ occurred during the first contact, subsequently decreasing to the intermediate value.

Towards the end of the trajectory, the decrease in rotation occurred at much shorter time intervals than the increase on steeper terrain. A typical example thereof is presented here, a relatively short ground contact at $70.81 \mathrm{~s}$, lasting $13 \mathrm{~ms}$. During this time, rotation decreased from $1^{\prime} 831$ to $1^{\prime} 539^{\circ} \mathrm{s}^{-1}$, reaching a local minimum of $1^{\prime} 458^{\circ} \mathrm{s}^{-1}$ in between. The 

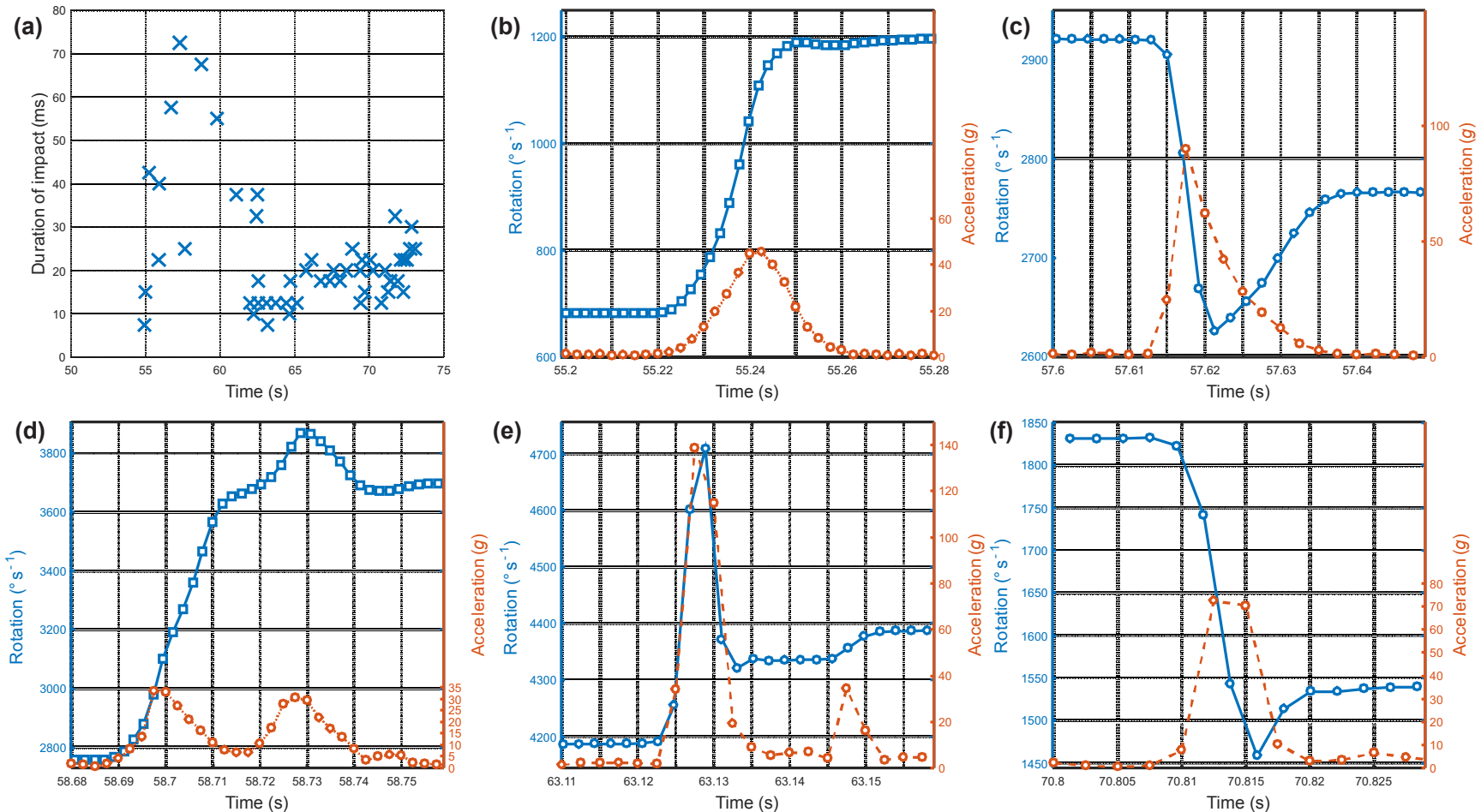

Figure 2. (a) Duration of ground contacts. (b) Absolute rotational and acceleration values for the ground contact at $55.24 \mathrm{~s}$ lasting $42 \mathrm{~ms}$, (c) at $57.62 \mathrm{~s}$ lasting $28 \mathrm{~ms}$, (d) at $58.72 \mathrm{~s}$ lasting $68 \mathrm{~ms}$, (e) a double contact from $63.13 \mathrm{~s}$ onward lasting $13 \mathrm{~ms}$ and $8 \mathrm{~ms}$, (f) and at $70.81 \mathrm{~s}$ lasting $13 \mathrm{~ms}$. The error on individual measurements is smaller than the plotted marker size.

maximum acceleration for this ground contact was $72.5 \mathrm{~g}$ (Fig. 2f).

\section{Discussion}

Because the acceleration and rotation sensors exhibit very small inherent offsets, a correction is not mandatory - but feasible if desired. The constant offsets being smaller than $0.1 \%$ for the gyroscope and $0.8 \%$ for the accelerometer with respect to the full range capacity of each individual sensor undermines the high-quality sensor stream. An evaluation of the sensor's centrical installation in the block indicated a very small eccentricity of $4 \mathrm{~mm}$. This shows that a careful manual placement is sufficient for accurate results.

In this experiment, ground contact duration was shown to vary considerably, ranging from a minimum of $8 \mathrm{~ms}$ to a maximum of $75 \mathrm{~ms}$. These measurements show that longer contacts occurred on steeper terrain and shorter ones on flatter terrain. However, no precise characterization is possible yet because the spatial data cannot be linked to the temporal data within the needed accuracy.

Temporal information on the block's flight duration between ground contacts can be used to calculate the jump height of the flight parabola (Gerber, 2015). A temporal and/or spatial link could be used to calculate the jump distance on the slope, but no such link has been established yet.
The measurements suggest very different forms of contact, both in terms of acceleration and rotation. For very short contacts of less than $10 \mathrm{~ms}$, the individual measurements are not quite as reliable as the quality control purports. To measure such short contacts, the measurement frequency would have to be increased, which is achieved by updating to StoneNode v1.1, which has an increased sampling rate of $1 \mathrm{kHz}$ for the accelerometer and gyroscope (Caviezel et al., 2018c).

\section{Conclusions}

These measurements show that high-quality, detailed and reliable analyses of rotational and acceleration data for rocks hitting the ground are possible. The applied sensors and measurement techniques provide a logistically simple but effective tool to obtain kinematic data for falling rocks. The measured data provide insight into highly dynamic impact processes, but additionally raise new questions, primarily concerning the spatial relation of the rock to the surface of the terrain. For example, the rock's velocity vector at the onset of a contact relative to the slope of the surface should be known to evaluate the response of the ground material. Clearly the rock-based sensors must be combined with highresolution external remote-sensing methods such as photogrammetry, lidar or radar to obtain the needed information. Most of these techniques are well adapted and tuned 
to quasi-static conditions, that is difference mapping and/or long-term monitoring. Extending these time-of-flight measurement techniques to track a rockfall trajectory in real time fails to date due to insufficient range, resolution and/or frame rate capabilities (Horaud et al., 2016). Possible solutions are a high frame-rate, simultaneously triggered multi-camera setup and subsequent stereographic reconstruction of the trajectory, or a highly specified time-of-flight camera such as a scannerless lidar system capable of tracking motions as fast as $100 \mathrm{~km} \mathrm{~h}^{-1}$ in single-reflection mode over large distances. For experimental purposes, being interested in direct flight kinematics, these approaches might be favored over seismic signal analysis (Hibert et al., 2017; Dietze et al., 2017).

We have mainly gathered and processed temporal information from the rockfall sensors. A connection to the spatial extent of the trajectory is still missing. An approach involving the projected longitudinal profile is available, but the exact connection to the inclination of the terrain or the assignment of slopes to each ground contact is not yet possible. This information would provide a better explanation of the general increases and decreases in rock rotation.

The sensor data are ideal to calibrate constitutive relationships, which are at the kernel of the RAMMS rockfall software module (Caviezel et al., 2018c, b). The combination of real-terrain measurements coupled with non-smooth modeling approaches opens many new possibilities to investigate how terrain influences rock motion. Because terrain is seldom homogeneous and rock shapes far from symmetric, in situ measurements are needed to measure the forces at play at any given time and impact, but also for every possible rockfall trajectory. Simulated results can now be calibrated to measured data to provide a calibration methodology for rockfall simulation codes.

Data availability. Data are available via EnviDat (https://www. envidat.ch), the environmental data portal developed at the WSL. Under https://doi.org/10.16904/envidat.37 (Caviezel et al., 2018d) a data archive is found containing site-specific geographical data such as DSM and orthophotos as well as the deposition points of manually induced rockfall by releasing differently shaped boulders with $30-80 \mathrm{~kg}$ of mass. Additionally available are all the StoneNode v1.0 data streams for rocks equipped with a sensor. The scrutinized data stream presented here is labelled "EOTA_RF05_r6".

Author contributions. Both authors contributed equally to the conception of the experiment, the data analysis and the paper preparation.

Competing interests. The authors declare that they have no conflict of interest.

Edited by: Jean-Philippe Malet

Reviewed by: François Noël and one anonymous referee

\section{References}

Altshuler, E., Torres, H., Gonzáalez-Pita, A., Sánchez-Colina, G., Pérez-Penichet, C., Waitukaitis, S., and Hidalgo, R. C.: Settling into dry granular media in different gravities, Geophys. Res. Lett., 41, 3032-3037, 2014.

Asteriou, P., Saroglou, H., and Tsiambaos, G.: Geotechnical and kinematic parameters affecting the coefficients of restitution for rock fall analysis, Int. J. Rock Mech. Min. Sci., 54, 103-113, 2012.

Caviezel, A., Bühler, Y., Christen, M., and Bartelt, P.: Induced Rockfall Dataset (Small Rock Experimental Campaign), Tschamut, Grisons, Switzerland; WSL Institute for Snow and Avalanche Research SLF; https://doi.org/10.16904/envidat.37, 2018a.

Caviezel, A., Bühler, Y., Lu, G., Christen, M., and Bartelt, P.: Proceedings of the 9th European Conference on Numerical Methods in Geotechnical Engineering, 25-27 June, Porto (Portugal), 875886, 2018b.

Caviezel, A., Schaffner, M., Cavigelli, L., Niklaus, P., Bühler, Y., Bartelt, P., Magno, M., and Benini, L.: Design and Evaluation of a Low-Power Sensor Device for Induced Rockfall Experiments, IEEE T. Instrum. Meas., 67, 767-779, 2018c.

Caviezel, A., Bühler, Y., Christen, M., and Bartelt, P.: WSL Institute for Snow and Avalanche Research SLF, https://doi.org/10.16904/envidat.37, 2018d.

Corona, C., Lopez-Saez, J., Favillier, A., Mainieri, R., Eckert, N., Trappmann, D., Stoffel, M., Bourrier, F., and Berger, F.: Modeling rockfall frequency and bounce height from three-dimensional simulation process models and growth disturbances in submontane broadleaved trees, Geomorphology, 281, 66-77, 2017.

Dietze, M., Mohadjer, S., Turowski, J. M., Ehlers, T. A., and Hovius, N.: Seismic monitoring of small alpine rockfalls - validity, precision and limitations, Earth Surf. Dynam., 5, 653-668, 2017.

Gerber, W.: Geschwindigkeit und Energie aus der Anlayse von Steinschlagspuren - Velocity and kinetic energy from the analysis of rockfall trajectories, Österr. Ing.- Archit.-Z., 160, 171-175, 2015.

Gronz, O., Hiller, P. H., Wirtz, S., Becker, K., Iserloh, T., Seeger, M., Brings, C., Aberle, J., Casper, M. C., and Ries, J. B.: Smartstones: A small 9-axis sensor implanted in stones to track their movements, Catena, 142, 245-251, 2016.

Hibert, C., Malet, J.-P., Bourrier, F., Provost, F., Berger, F., Bornemann, P., Tardif, P., and Mermin, E.: Single-block rockfall dynamics inferred from seismic signal analysis, Earth Surf. Dynam., 5, 283-292, 2017.

Horaud, R., Hansard, M., Evangelidis, G., and Ménier, C.: An overview of depth cameras and range scanners based on time-offlight technologies, Mach. Vision Appl., 27, 1005-1020, 2016.

Leine, R., Schweizer, A., Christen, M., Glover, J., Bartelt, P., and Gerber, W.: Simulation of rockfall trajectories with consideration of rock shape, Multibody Syst. Dyn., 32, 241-271, 2014.

Niklaus, P., Birchler, T., Aebi, T., Schaffner, M., Cavigelli, L., Caviezel, A., Magno, M., and Benini, L.: StoneNode: A lowpower sensor device for induced rockfall experiments, in: 2017 IEEE Sensors Applications Symposium (SAS), 1-6, 2017.

Paronuzzi, P.: Rockfall-induced block propagation on a soil slope, northern Italy, Environ. Geol., 58, 1451-1466, 2009. 
Sanchez-Colina, G., Alonso-Llanes, L., Martinez, E., BatistaLeyva, A. J., Clement, C., Fliedner, C., Toussaint, R., and Altshuler, E.: Note: "Lock-in accelerometry" to follow sink dynamics in shaken granular matter, Review of Scientific Instruments, 85, 2014.

Saroglou, C., Asteriou, P., Zekkos, D., Tsiambaos, G., Clark, M., and Manousakis, J.: UAV-based mapping, back analysis and trajectory modeling of a coseismic rockfall in Lefkada island, Greece, Nat. Hazards Earth Syst. Sci., 18, 321-333, https://doi.org/10.5194/nhess-18-321-2018, 2018.
Trappmann, D. and Stoffel, M.: Visual dating of rockfall scars in Larix decidua trees, Geomorphology, 245, 62-72, 2015. 